\title{
Energy-windowed, pixellated X-ray diffraction using the Pixirad CdTe detector
}

\author{
D. O’Flynn, ${ }^{a}$ R. Bellazzini, ${ }^{b}$ M. Minuti, ${ }^{b}$ A. Brez, ${ }^{b}$ M. Pinchera, ${ }^{b}$ G. Spandre, ${ }^{b}$ R. Moss ${ }^{a}$ and \\ R.D. Speller ${ }^{a}$ \\ ${ }^{a}$ Department of Medical Physics and Biomedical Engineering, University College London, Gower Street, \\ London WC1E 6BT, UK \\ ${ }^{b}$ INFN Pisa and Pixirad Imaging Counters srl, Largo Pontecorvo 3, 56127 Pisa, Italy \\ E-mail: d.oflynn@ucl.ac.uk
}

AвSTRACT: X-ray diffraction (XRD) is a powerful tool for material identification. In order to interpret XRD data, knowledge is required of the scattering angles and energies of X-rays which interact with the sample. By using a pixellated, energy-resolving detector, this knowledge can be gained when using a spectrum of unfiltered X-rays, and without the need to collimate the scattered radiation. Here we present results of XRD measurements taken with the Pixirad detector and a laboratorybased X-ray source. The cadmium telluride sensor allows energy windows to be selected, and the $62 \mu \mathrm{m}$ pixel pitch enables accurate spatial information to be preserved for XRD measurements, in addition to the ability to take high resolution radiographic images. Diffraction data are presented for a variety of samples to demonstrate the capability of the technique for materials discrimination in laboratory, security and pharmaceutical environments. Distinct diffraction patterns were obtained, from which details on the molecular structures of the items under study were determined.

KEYwords: Detection of contraband and drugs, detection of explosives, inspection with X-rays

\footnotetext{
${ }^{1}$ Corresponding author.
} 


\section{Contents}

1 Introduction 1

2 Experimental detail 3

3 Results 4

4 Conclusion $\quad 8$

\section{Introduction}

X-ray diffraction (XRD) occurs when X-rays are coherently scattered from the molecular layers of a material and subsequently constructively interfere. For a molecular layer spacing of $d$, the scattering angles $2 \theta$ at which this interference can be seen are given by Bragg's law:

$$
n \lambda=2 d \sin \theta
$$

where $\lambda$ is the X-ray wavelength. The nature of the interference pattern gives detailed information on molecular structure, and thus is a highly useful technique for materials identification, giving complementary information to a traditional X-ray transmission or dual-energy image [1-3]. XRD is a non-destructive method, and the ability of X-rays to pass through barriers gives an advantage over other molecular analysis methods in cases where the materials of interest are obscured, for example when samples are inside packaging, or in security scenarios. In order to retrieve the X-ray interference information, both the energy (wavelength) and the scattering angle of the X-rays must be well defined. This can be achieved either by fixing the energy and measuring the angular dependency of the scattered radiation (angular dispersive XRD or ADXRD) or by fixing the scattering angle and measuring the energy dependency or the scattered radiation (EDXRD) [4-6].

In the case of ADXRD, the X-ray energy can be defined by the use of a synchrotron or, in the laboratory, by filtering a standard X-ray source. The scattering angle is defined by collimating the incident and scattered X-rays, and the angular dependency is observed by a rotation of the X-ray source and detector about the sample; alternatively the uncollimated scattered field can be measured with a pixellated, area sensitive sensor. For EDXRD, the incident and scattered X-rays are also collimated, but the geometry is kept fixed. A broad X-ray spectrum is incident upon the sample, and an energy-resolving detector is used to isolate energy windows and produce XRD data. Recently, a pixellated diffraction (PixD) method which combines features of both ADXRD and EDXRD was developed, allowing the use of a polychromatic X-ray source and uncollimated scattered X-rays to be detected, giving a large increase in counting efficiency $[7,8]$. This technique utilised the pixellated, energy-resolving HEXITEC detector [9-11], which enabled both spatial and spectral diffraction information to be preserved simultaneously. 
In this work, a similar experimental approach to PixD was applied using the Pixirad PIXI-III detector [12-15]. The Pixirad detector has an area of $31.7 \times 25.0 \mathrm{~mm}^{2}$, containing a $512 \times 402$ pixel array with $62 \mu \mathrm{m}$ pitch. The version of the detector used for this work has a $750 \mu \mathrm{m}$ thick cadmium telluride (CdTe) X-ray sensor, and is capable of performing energy windowing with user-defined limits. The CdTe has a very high detection efficiency at this thickness for X-ray energies under $100 \mathrm{keV}$, and, unlike germanium-based detectors, CdTe-based detectors can be operated without the requirement of cooling to cryogenic temperatures. The energy windowing is achieved by setting thresholds below which all photon events are discarded; if two such thresholds are set at different energies then their difference represents the detected photons with energies between the two thresholds. Photons with energy falling within the selected window are counted directly in the ASIC pixels, so that only one reading is required to get the windowed counts. The energy resolution of the detector is approximately $4 \mathrm{keV}$ at $60 \mathrm{keV}$. An advantage of this energy windowing approach is the ability to select an energy region of interest, rather than being limited by the emission lines of the X-ray spectrum (as is the case in a typical diffractometer). A practical application of this technique is the selection of an energy high enough for transmission XRD measurements through samples of several millimetres thickness. Additionally, since the energy selection is performed by the detector itself, the issue of motion-sensitive monochromator alignment is negated.

It is shown here that by using the Pixirad detector to measure scattered X-rays within a selected energy window, pixellated diffraction information can be preserved with the use of a polychromatic incident X-ray beam and no collimation between the sample and detector. Diffraction data for a variety of samples is presented to demonstrate the material specificity of the PixD technique using Pixirad. The small pixel size of the detector, and its ability to record a primary beam flux is demonstrated with an image of a packet of paracetamol tablets; the diffraction data for one of these tablets is also presented.

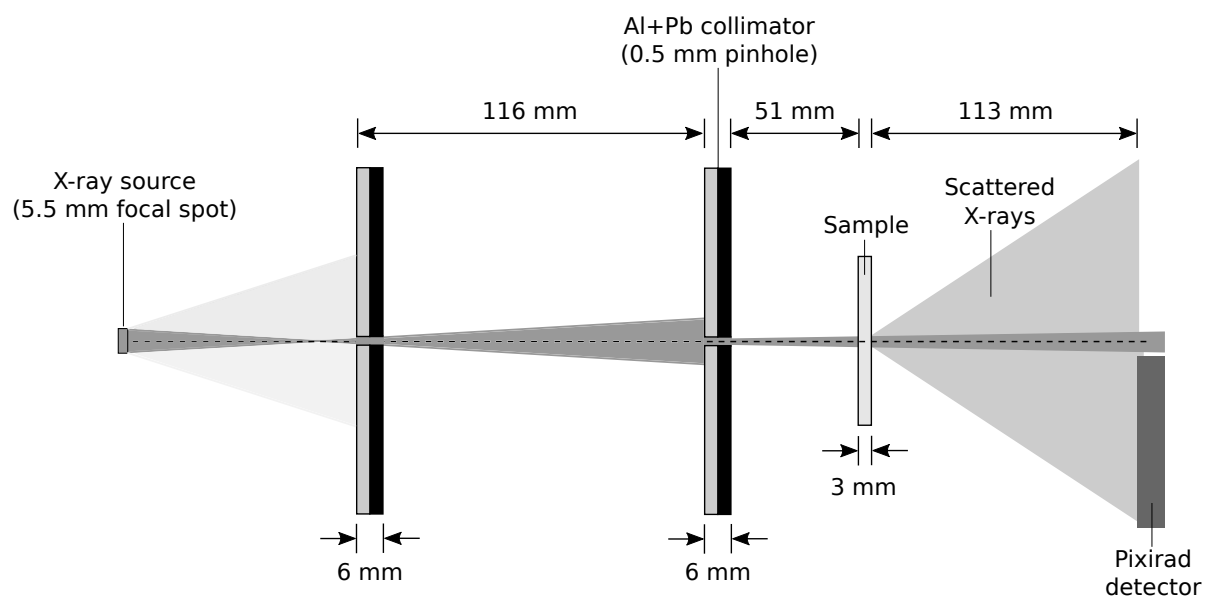

Figure 1. Schematic diagram of the experimental setup used for the pixellated diffraction measurements in Figures 4 and 5. 


\section{Experimental detail}

Initial measurements to test the energy-windowing capability of the detector were carried out with the X-ray beam aligned with the centre of the detector, and the tube operated at $40 \mathrm{keV}$. A schematic of the experimental setup for subsequent measurements on all samples is shown in Figure 1. The X-ray source used for these measurements was a Thales THX 1601055 with a $5.5 \mathrm{~mm}$ focal spot size, operated at $56 \mathrm{kV}$ and $6.0 \mathrm{~mA}$. The incident X-rays were collimated by two lead sheets of $3 \mathrm{~mm}$ thickness each with a $0.5 \mathrm{~mm}$ diameter hole, separated by $116 \mathrm{~mm}$, to give a pencil beam of approximately $1 \mathrm{~mm}$ diameter at the sample. For all measurements the Pixirad detector was positioned behind the sample, such that diffraction measurements were taken in transmission mode. The detector was operated at a temperature of $-30{ }^{\circ} \mathrm{C}$, with a voltage of $600 \mathrm{~V}$ applied to the CdTe sensor. The primary X-ray beam was positioned $2 \mathrm{~mm}$ outside the corner of the detector to ensure that only scattered photons were detected. Background scatter with no sample in place was separately recorded, and subsequently subtracted from the sample diffraction data. Diffraction data were collected with an energy window of $22-24.5 \mathrm{keV}$. This energy window was selected to show diffraction across the face of the detector based on the geometry of the system, the incident X-ray spectrum, and the intermolecular spacings of interest for the samples under study. Each pixel of the detector is sensitive to a particular scattering angle due to the geometry of the system, neglecting the effects of sample thickness [16]. Taking the centre of the selected energy window, a conversion to momentum transfer $(x)$ space was made for each pixel using the relation

$$
x=\frac{1}{\lambda} \sin \theta
$$

The momentum transfer coverage across the detector face is shown in Figure 2(a) and the weighting of momentum transfer sensitivity (i.e. how many pixels cover each region of the scattering angle range) is shown in Figure 2(b).

The samples studied for this work were caffeine and hexamine powder; plastic explosive samples C4, Semtex 1A and Semtex H; a sheet of aluminium and a paracetamol tablet both outside and inside its packaging. This range of materials have the common feature of containing components
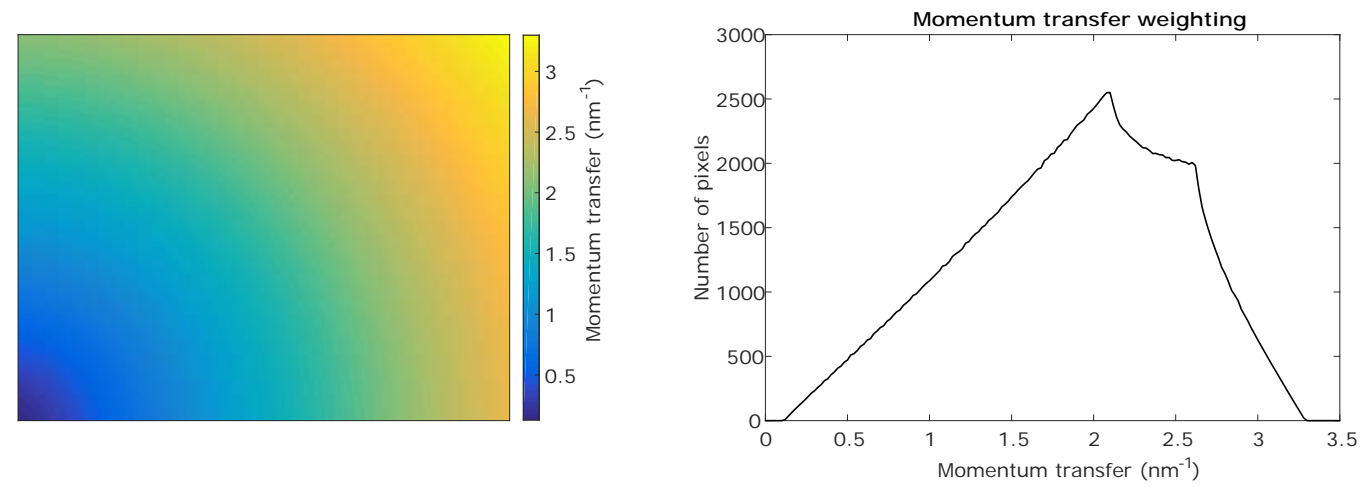

Figure 2. (a) Momentum transfer coverage of the detector face based on the scattering angles observed at each pixel due to the experimental geometry and an X-ray photon energy of $23.25 \mathrm{keV}$ - the centre of the 22-24.5 keV energy window. (b) The momentum transfer weighting which arises due to the experimental setup. 

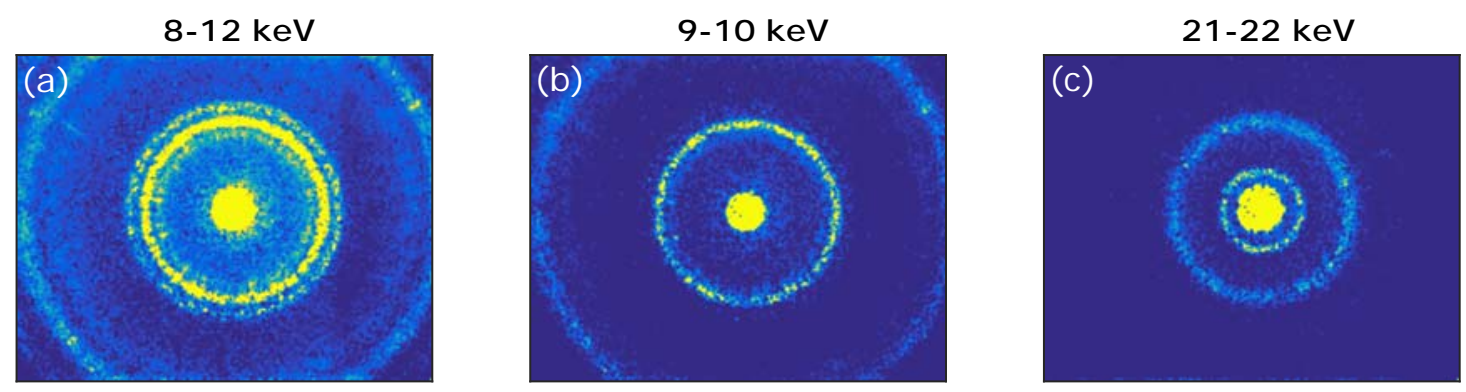

$12-40 \mathrm{keV}$

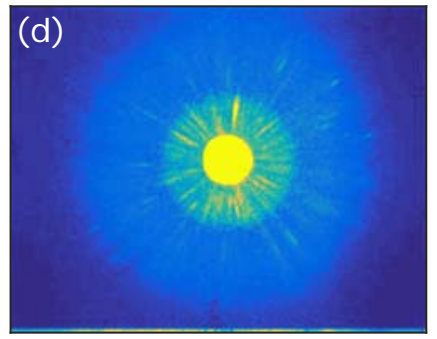

22-40 keV

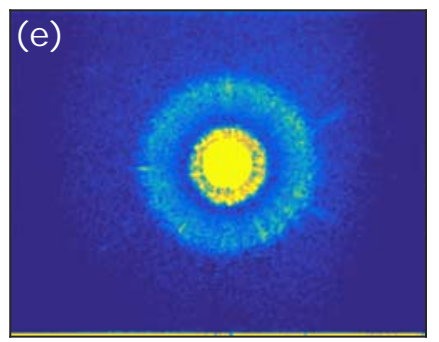

Figure 3. Preliminary experiments demonstrating the energy-windowing capability of Pixirad for recording diffraction data from a polycrystalline caffeine sample. The bright central spot is due to the incident X-ray beam.

with regular crystal/molecular ordering (and thus give diffraction patterns from which the ordering can be determined), whilst being in distinct physical forms. In particular, the explosives and the paracetamol were selected so as to demonstrate the potential for this technique to non-destructively identify materials of interest which are often obscured by barriers and thus cannot be examined by conventional methods without physically removing these barriers. Each sample was $3 \mathrm{~mm}$ thick, with the exception of the $3.5 \mathrm{~mm}$ paracetamol tablet. The thicknesses used gave a compromise between the amount of XRD signal, and the X-ray attenuation and broadening of diffraction peaks seen for thicker samples.

For the transmission image of the paracetamol packet, all collimation was removed from the system to give complete beam coverage of the detector. The packet was placed within $5 \mathrm{~mm}$ of the detector to mitigate blurring due to the large focal spot size. The image presented is a composite $4 \times 4$ grid; each frame was acquired with a $117 \mathrm{~ms}$ exposure with X-ray tube settings of $56 \mathrm{kV}$ and $0.3 \mathrm{~mA}$, with an energy window of $18-56 \mathrm{keV}$, and flat-field corrected.

\section{Results}

Initial characterisation measurements were taken of a polycrystalline caffeine sample, with the collimated X-ray beam aligned with the centre of the detector. As described by Bragg's law, the diffraction can be seen to spread radially from the primary beam position, along lines of constant angle. When an energy window of $8-12 \mathrm{keV}$ is selected, the two expected diffraction rings from the caffeine can be seen $[17,18]$, although rather than two distinct rings, there appears to be a triplet effect on each ring (Figure 3(a)). These additional rings were assumed to be a result of the 

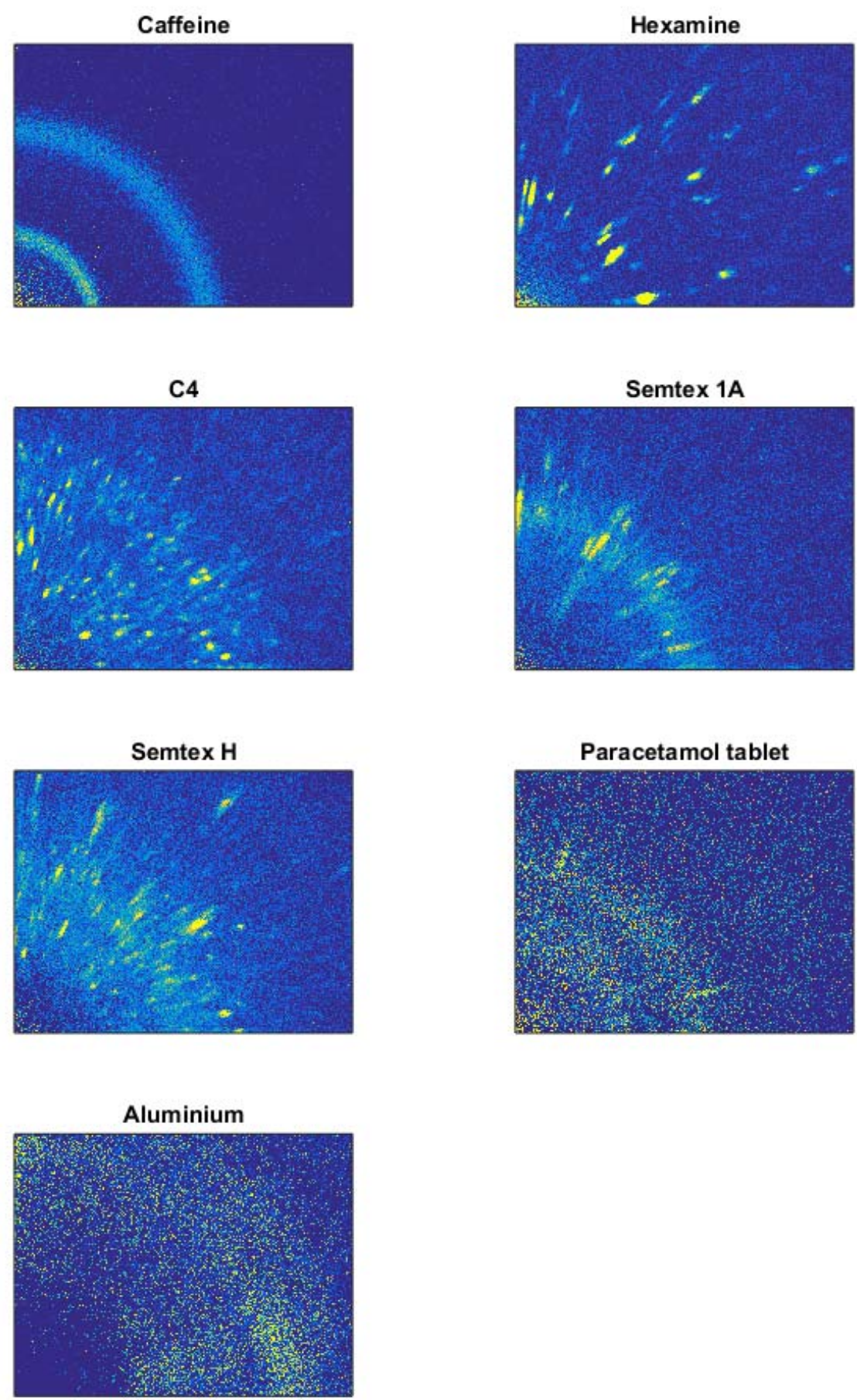

Figure 4. Pixellated diffraction patterns recorded for each sample with X-ray tube settings of $56 \mathrm{kV}$ and $6.0 \mathrm{~mA}$, and an energy window of $22-24.5 \mathrm{keV}$. The acquisition times used were $4425 \mathrm{~s}$ for caffeine, $600 \mathrm{~s}$ for hexamine, C4, Semtex 1A and Semtex H, and 60 s for paracetamol and aluminium. The data are corrected for a background reading taken with no sample in place and an acquisition time of $3600 \mathrm{~s}$. 

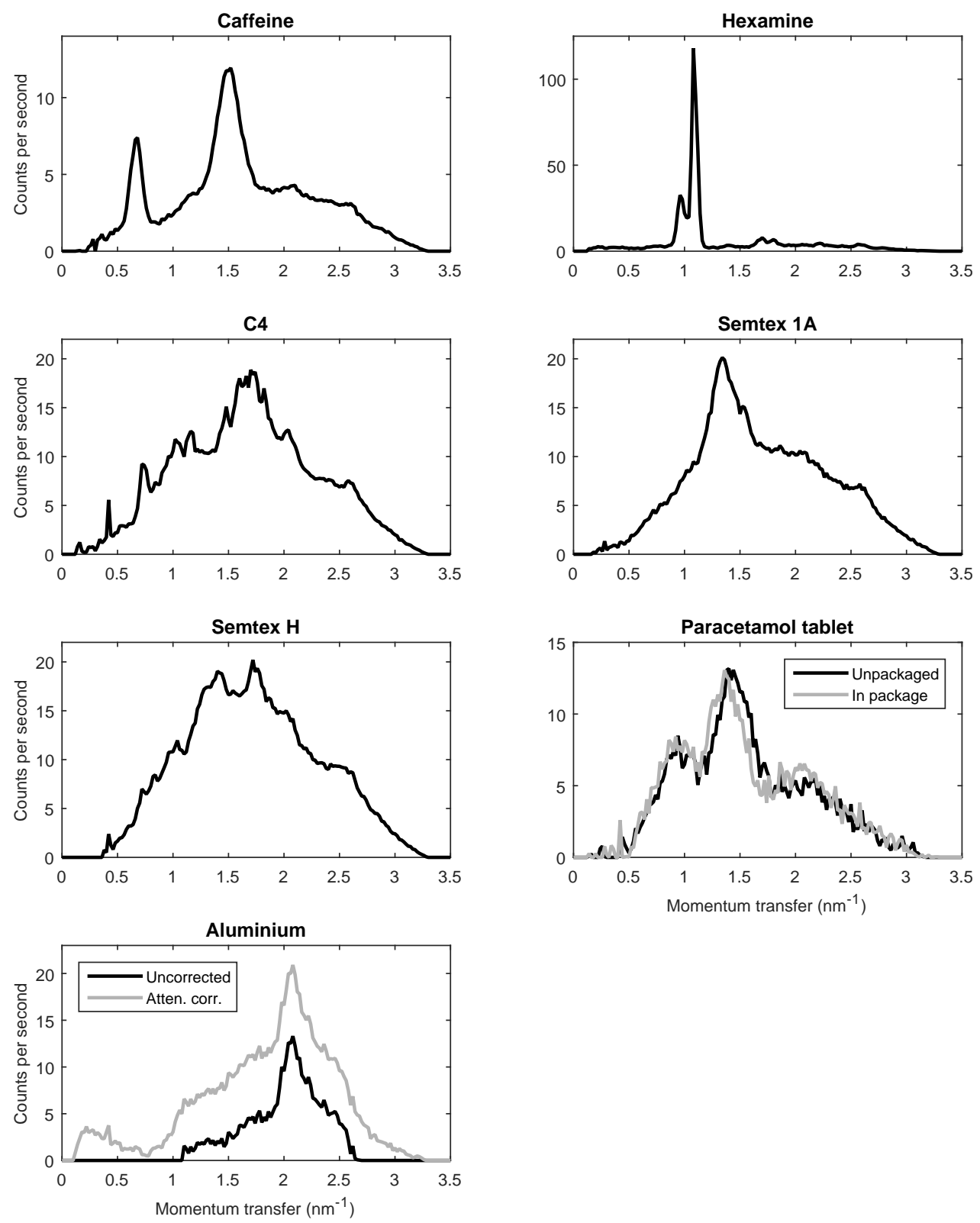

Figure 5. Momentum transfer plots as calculated from the diffraction data shown in Figure 3. Paracetamol data are shown for the tablet both outside and inside the blister packaging (made up of aluminium foil and plastic). Attenuation corrected data is also given for the aluminium sample. 
relatively strong characteristic L-alpha, L-beta and L-gamma tungsten emissions from the X-ray source at energies of $8.34 \mathrm{keV}, 9.96 \mathrm{keV}$ and $11.28 \mathrm{keV}$, respectively. In order to mitigate this tripling of the diffraction rings, another measurement was taken with a narrower energy window of 9-10 keV. In this case, the contribution of the L-alpha and L-gamma X-ray lines are not recorded, as can be seen in the diffraction pattern in Figure 3(b). A confirmation of the energy windowing for a different energy range of 21-22 keV is given in Figure 3(c), where it can be seen that the diffraction rings are decreasing in radius (i.e. decreased scattering angle), as expected from Bragg's law. A demonstration of the necessity for this strict energy windowing for diffraction measurements is shown in Figures 3(d) and 3(e), where wide energy ranges of 12-40 keV and 22-40 keV result in the sharp diffraction rings becoming blurred out due to the sensitivity to diffraction across the Bremsstrahlung continuum.

Further measurements of energy-windowed pixellated diffraction were taken with the experimental setup as shown in Figure 1. The diffraction patterns recorded for seven different samples are shown in Figure 4. The two Debye-Scherrer ring sections seen for caffeine represent constructive interference of X-rays in the 22-24.5 keV energy range from caffeine $d$-spacings of $7.53 \AA, 7.40 \AA$, $3.37 \AA$ and $3.30 \AA$. The first two and latter two spacings are not individually resolved [8]. Rings are seen due to the fact that the caffeine sample was a fine powder, and as such the primary X-ray beam was incident upon a large number of crystallites with random orientations about the incident beam axis. Converting this data to momentum transfer space, the rings are seen as two peaks in intensity (Figure 5). The position of these peaks can be used to infer inter-molecular spacings by a combination of equations 1.1 and 2.1 to give $d=1 / 2 x$. The observed $d$-spacings are in agreement with those found in the literature $[17,18]$.

The larger grain hexamine sample instead shows diffraction in the form of spots arranged radially. The corresponding momentum transfer plot shows sharp peaks of much greater intensity than those for caffeine, and appears more similar to diffraction data from a single crystal sample. In this case, the hexamine crystallites were approximately $1 \mathrm{~mm}$ in diameter, of a comparable size to that of the X-ray beam. The two primary peaks in intensity at $0.96 \mathrm{~nm}^{-1}$ and $1.08 \mathrm{~nm}^{-1}$ correspond to $d$-spacings of $5.21 \AA$ and $4.63 \AA$, respectively.

The three plastic explosive compounds contain different combinations of explosive compounds; C4 contains RDX, Semtex 1A contains PETN, and Semtex H contains a mixture of both RDX and PETN. The nature of their respective pixellated diffraction images in Figure 4 implies a granular nature to these compounds with grain sizes likely larger than those of the caffeine sample but smaller than those of the hexamine. The momentum transfer spectrum for Semtex H shows features which are individually seen in those of $\mathrm{C} 4$ and Semtex $1 \mathrm{~A}$, due to the presence of both RDX and PETN (specifically, multiple RDX peaks at $0.42 \mathrm{~nm}^{-1}, 0.72 \mathrm{~nm}^{-1}, 1.02 \mathrm{~nm}^{-1}$; and the main PETN peak at $\left.1.36 \mathrm{~nm}^{-1}\right)$.

The features seen in the diffraction data for the paracetamol tablet in Figure 2 correspond with the crystal structure of Form I paracetamol, notably the smaller peak at $0.9 \mathrm{~nm}^{-1}$ ( $d$-spacings of $0.53 \AA$ and $0.57 \AA$ ) and the broader peak centred at $1.4 \mathrm{~nm}^{-1}$ ( $d$-spacings of $0.34 \AA, 0.36 \AA$ and $0.38 \AA$ ) [19]. In order to show the capability of this XRD technique to probe molecular structure through barriers, the diffraction data of the paracetamol tablet inside the blister packet is also shown in Figure 2. There is a slight increase in signal at $2.1 \mathrm{~nm}^{-1}$ which is believed to be due to diffraction from the aluminium foil present in the packaging. The slight shift in momentum transfer of the 


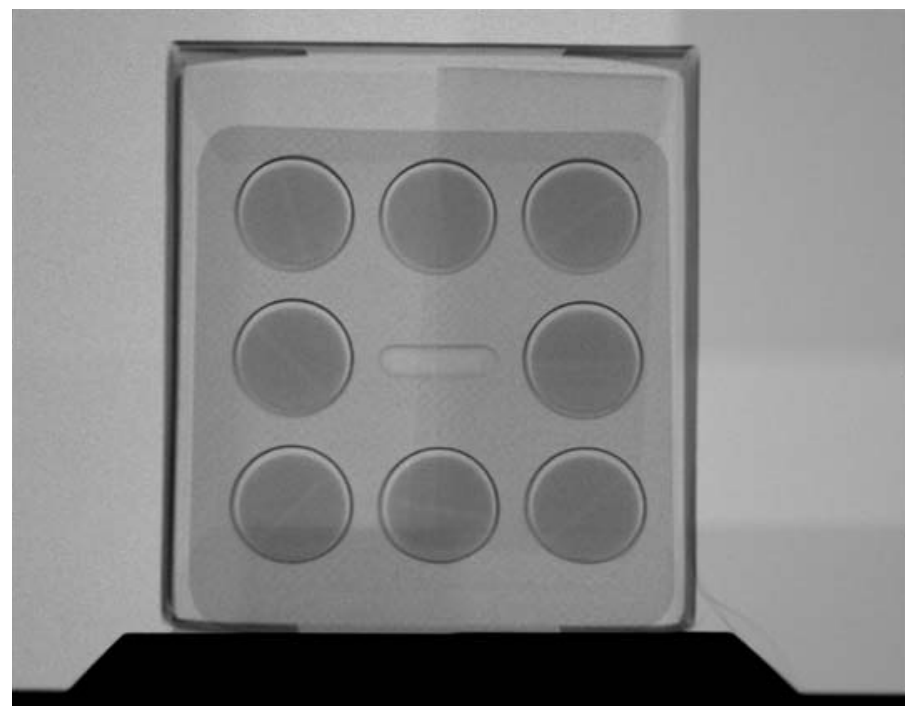

Figure 6. Radiographic image of the paracetamol packet inside the box, taken with the Pixirad detector. The figure is a $4 \times 4$ stitched image, with each constituent image being acquired for $117 \mathrm{~ms}$, with X-ray tube settings of $56 \mathrm{kV}$ and $0.5 \mathrm{~mA}$, and an energy window of $18-56 \mathrm{keV}$

main peak is likely due to a slight error in sample positioning (of the order of $1 \mathrm{~mm}$ ). The imaging capability of the Pixirad detector is demonstrated for the paracetamol tablets inside the blister packaging and box in Figure 6.

The previously reported XRD data for aluminium show peaks corresponding to $2.11 \mathrm{~nm}^{-1}$, $2.48 \mathrm{~nm}^{-1}, 3.48 \mathrm{~nm}^{-1}$, and $4.07 \mathrm{~nm}^{-1}$ - the (111), (200), (220) and (311) reflections, respectively [20]. In the pixellated diffraction data presented here, the (111) peak is clearly seen, although the latter three are outside the momentum transfer range of the measurement - with the possible exception of a shoulder in the data at $2.5 \mathrm{~nm}^{-1}$. The tailing off of the diffraction spectrum for aluminium occurs at a lower momentum transfer value than for the other samples $\left(\sim 2.6 \mathrm{~nm}^{-1}\right.$ compared with $\left.3.2 \mathrm{~nm}^{-1}\right)$. This is assumed to be due to the additional attenuation from the relatively high- $Z /$ density material for photons in the $22-24.5 \mathrm{keV}$ range, which is enhanced when scattering at higher angles (i.e. higher momentum transfers) since more material is in the beam path on average.

A correction for the aluminium attenuation was made by rescaling the background data before subtraction by a factor of $\exp \left(-\mu_{E} t\right)$, where $t$ is the thickness of the aluminium plate $(0.3 \mathrm{~cm})$ and $\mu_{E}$ is the linear attenuation coefficient of aluminium at $23.25 \mathrm{keV}\left(5.988 \mathrm{~cm}^{-1}\right)$. The value of $\mu_{E}$ was obtained from the XCOM database [21] using an aluminium density of $2.7 \mathrm{~g} \mathrm{~cm}^{-3}$.

\section{Conclusion}

This work has demonstrated the capability of the Pixirad detector to measure a pixellated diffraction signal from an unfiltered, polychromatic X-ray source, giving a through-barrier probe of molecular structure. The CdTe detector material has a high detection efficiency across the energy range of interest for this application, and the readout electronics of Pixirad give rise to energy resolution and the ability to correct for charge-sharing effects. These factors combine to give the capability to select a user-defined energy window of sensitivity. Without energy-windowing, the coherent diffraction 
signal would be spread out spatially across the detector due to the X-ray emission spectrum, and prominent features relating to crystal or molecular structures would be lost. Alternatively, if the incident beam was filtered to be monoenergetic, a large reduction in X-ray flux, and thus poorer counting statistics, would have resulted. The spatial resolution afforded by the detector pixels enables angular scatter information to be preserved without the need for collimation of the scattered beam - this is true provided the sample thickness remains of the order of several millimetres [16] - and as such the counting statistics of the system are greatly improved with respect to previous EDXRD systems based on a single-element detector. Additionally, further information on the grain size present in the sample is preserved with the diffraction image, as can be seen by comparing the Debye-Scherrer rings in fine grain caffeine and distinct spots in large grain hexamine [7,8]. The peak widths for this diffraction technique are wider than those in diffractometer scans. The main factors contributing to the peak broadening are uncertainties in the geometry of the system (due to incident beam size and divergence, and sample thickness), and the energy resolution of the detector.

Due to the small pixel pitch of the Pixirad detector, it can also be used to take high-quality radiographic images. The option of energy-windowing gives additional potential applications for complementary multispectral imaging and XRD measurements using the same detector. The XRD experiments performed in this work required a collimated incident beam, it would be timeconsuming to scan an entire sample in this manner. A practically implemented materials identification system could involve a system where an initial X-ray image of an object is taken, from which regions of interest could be identified for further analysis with XRD.

\section{Acknowledgements}

This research was funded under the Innovative Research Call in Explosives and Weapons Detection (2010) initiative, a cross-government programme sponsored by a number of departments and agencies under the UK Government's CONTEST strategy, in partnership with US Department of Homeland Security (IRC2010/ITT030). The explosive samples were provided by the UK Home

Office Centre for Applied Science and Technology. The experiments and handling of these samples were conducted in full compliance with UK laws, rules and regulations.

\section{References}

[1] Shell J W 1963 Journal of Pharmaceutical Sciences 52 24-29 ISSN 1520-6017

[2] Peplow D E and Verghese K 1998 Physics in Medicine and Biology 432431

[3] Harding G and Schreiber B 1999 Radiation Physics and Chemistry 56229 - 245 ISSN 0969-806X

[4] Kämpfe B, Luczak F and Michel B 2005 Particle \& Particle Systems Characterization 22 391-396 ISSN 1521-4117

[5] Cook E, Fong R, Horrocks J, Wilkinson D and Speller R 2007 Applied Radiation and Isotopes 65959 - 967 ISSN 0969-8043

[6] Cook E, Pani S, George L, Hardwick S, Horrocks J and Speller R 2009 IEEE Transactions on Nuclear Science 56 1459-1464 ISSN 0018-9499

[7] O’Flynn D, Reid C B, Christodoulou C, Wilson M D, Veale M C, Seller P, Hills D, Desai H, Wong B and Speller R 2013 Journal of Instrumentation 8 P03007 
[8] O’Flynn D, Crews C, Drakos I, Christodoulou C, Wilson M D, Veale M C, Seller P and Speller R D 2016 Journal of Physics D: Applied Physics 49175304 URL

http: //stacks.iop.org/0022-3727/49/i=17/a=175304

[9] Jones L, Seller P, Wilson M and Hardie A 2009 Nuclear Instruments and Methods in Physics Research Section A: Accelerators, Spectrometers, Detectors and Associated Equipment 60434 - 37 ISSN 0168-9002 Proceedings of the 8th International Conference on Position Sensitive Detectors

[10] Seller P, Bell S, Cernik R J, Christodoulou C, Egan C K, Gaskin J A, Jacques S, Pani S, Ramsey B D, Reid C, Sellin P J, Scuffham J W, Speller R D, Wilson M D and Veale M C 2011 Journal of Instrumentation $6 \mathrm{C} 12009$

[11] Veale M C, Kalliopuska J, Pohjonen H, Andersson H, Nenonen S, Seller P and Wilson M D 2012 Journal of Instrumentation $7 \mathrm{C} 01035$

[12] Bellazzini R, Brez A, Spandre G, Minuti M, Pinchera M, Delogu P, de Ruvo P and Vincenzi A 2015 Journal of Instrumentation $10 \mathrm{C} 01032 \mathrm{URL}$ http : //stacks . iop.org/1748-0221/10/i=01/a=C01032

[13] Romano A, Pacella D, Claps G, Causa F and Gabellieri L 2015 Journal of Instrumentation 10 C02046

[14] Vincenzi A, de Ruvo P, Delogu P, Bellazzini R, Brez A, Minuti M, Pinchera M and Spandre G 2015 Journal of Instrumentation 10 C04010

[15] Delogu P, Oliva P, Bellazzini R, Brez A, de Ruvo P, Minuti M, Pinchera M, Spandre G and Vincenzi A 2016 Journal of Instrumentation 11 P01015 URL http : //stacks . iop.org/1748-0221/11/i=01/a=P01015

[16] O’Flynn D, Desai H, Reid C B, Christodoulou C, Wilson M D, Veale M C, Seller P, Hills D, Wong B and Speller R D 2013 Crime Science 24

[17] Phadnis N V, Cavatur R K and Suryanarayanan R 1997 Journal of Pharmaceutical and Biomedical Analysis 15929 - 943 ISSN 0731-7085

[18] Lehmann C and Stowasser F 2007 Chemistry Ü A European Journal 13 2908-2911 ISSN 1521-3765

[19] Perrin M A, Neumann M A, Elmaleh H and Zaske L 2009 Chem. Commun. 22(22) 3181-3183

[20] Lei X f and Ma J x 2010 Journal of the Brazilian Chemical Society 21209 - 213 ISSN 0103-5053

[21] Berger M, Hubbell J, Seltzer S, Chang J, Coursey J, Sukumar R, Zucker D and Olsen K 2010 Xcom: Photon cross section database (version 1.5). [online] available: http://physics.nist.gov/xcom (viewed 30 September 2016) 